\title{
Innovations in
}

Insurance, Risk- and Asset Management

\section{Kathrin Glau}

Daniël Linders

Aleksey Min

Matthias Scherer

Lorenz Schneider

Rudi Zagst

editors 


\title{
Chapter 15
}

\section{Chance-Risk Classification of Pension Products: Scientific Concepts and Challenges}

\author{
Ralf Korn \\ Dept. Mathematics, Univ. Kaiserslautern, \\ Kaiserslautern, 67663, Germany \\ and \\ Dept. Financial Mathematics, Fraunhofer ITWM, \\ Kaiserslautern, 67663, Germany \\ korn@mathematik.uni-kl.de \\ Andreas Wagner \\ Dept. Mathematics Group, Fraunhofer ITWM, \\ Kaiserslautern, 67663, Germany \\ andreas.wagner@itwm.fraunhofer.de
}

\begin{abstract}
We survey the underlying scientific concepts and aspects of the implementation of the classification of state-subsidized private German pension products into five different chance-risk classes. The topics range from the choice and calibration of the capital market model via simulation issues of various pension products to specific research topics such as the behavior of chance-risk curves or new valuation algorithms for cliquet-type options.
\end{abstract}

Keywords: chance-risk classification, pension products, Monte Carlo simulation.

\section{Introduction}

The introduction of bonus schemes (such as the Riester Rente) or schemes that lead to tax advantages (such as the Basis Rente) in Germany had the clear intention to give the public an incentive to enter into private pension contracts as a necessary add-on to their state pension. As understanding general pension products and judging their risk and return potential usually requires deep actuarial knowledge, the German Ministry of Finance introduced a new standardized information document (Produktinformationsblatt $)^{1}$ for those products to help the customer in comparing the

\footnotetext{
${ }^{1}$ Similar to the KID in the European PRIIPS regulation.

Open Access chapter published by World Scientific Publishing Company and distributed under the terms of the Creative Commons Attribution-NonCommercial-NoDerivatives (CC BY-NC 4.0) License.
} 
products and finding a suitable choice. Besides some product and concept descriptions, the leaflet has to contain two key figures

- a so-called chance-risk class ranging from 1 to 5 expressing the character of the chances and risks associated with the product,

- the reduction in yield expressing that part of the potential yield of the product that is lost due to costs charged by the issuing company (such as administration costs, costs of sales and management, or costs of capital or of investment).

It is required that the chance-risk classification has to be based on stochastic simulations of the evolution of the contract value of a product up to the end of the accumulation period. A chance-risk class of 1 is characterized by a low risk and a low return potential of the product, i.e. the customer should have a precise idea about the available money at the end of the accumulation period. In addition, the contract value has to increase over time, and there is a money-back-guarantee at the end of the accumulation period. A product having been assigned the chance-risk class 5 shows a high return potential, but also admits a high risk. Its capital at the end of the accumulation period is not required to stay above a positive bound. The classes 2, 3, 4 fill the space between 1 and 5 in increasing order of return potential and riskiness. Of those, only chance-risk class 2 in addition contains a money-back-guarantee. More details will be found in the sections below.

We will in the following only consider aspects of the chance-risk classification and do not comment on the concept and the computation of the reduction in yield. ${ }^{2}$

As the chance-risk classification has to be done by an independent organization, the Fraunhofer Institute for Industrial Mathematics ITWM in Kaiserslautern was chosen as the result of a public competition. A separate legal entity was formed, the Produktinformationsstelle Altersvorsorge $g G m b H$ which is responsible for the classification of the products. However, all mathematical concepts underlying the whole classification process have been developed by the Financial Mathematics Department of Fraunhofer ITWM. These include suggestions for

- the form of the capital market model underlying the simulation of the contract values at the end of the accumulation period,

\footnotetext{
${ }^{2}$ To learn more about the concept and the actual computation of the reduction in yield see www.produktinformationsstelle.de/effektivkosten.html.
} 
- the choice of the risk measure and the chance measure that form the basis for the chance-risk-classification of the pension products,

- the determination of the five different chance-risk classes,

- the simulation algorithms to obtain the contract value at the end of the accumulation period for the different pension products,

- the algorithms for calculating the reduction in yield for the different pension product types,

where the Ministry of Finance of Germany had the final say about accepting these suggestions.

We will in the following present the main conceptual ideas behind this project in general and will in particular concentrate on issues of financial mathematics and simulation. Let us also state in this introductory section that all the classifications have to be done for four different times until the end of the accumulation phase $T$ of the product in years with

$$
T \in\{12,20,30,40\} .
$$

This does not only result in an enormous simulation effort for just one product, but also leads to the possibility that one product can have four different chance-risk classes for the four different maturities. However, given that the product parameters (in particular with regard to its assigned costs) do not change dramatically with the maturities, the scenario of having different classifications for different maturities is not very likely. The extreme case of four different classes is extremely unlikely.

Of course, it would be desirable to be able to classify products of exactly that form that is tailored to the customer's personal needs. However, the resulting variety of products and of needed simulation and classification work cannot be delivered, at least not without exploding classification costs. As a compromise, the classification is only done for a prototypical customer who is assumed to pay a monthly contribution (before costs) of 100 Euros (where state bonuses are assumed to be included when they are granted) until the last month of the accumulation products, i.e. we assume $12 \cdot T$ payments. Further, we perform 10000 simulations of the wealth at the end of the accumulation period with a monthly time discretization as the basis for determining the chance and the risk measure.

We will in the following present

- the main types of private pension products offered in Germany,

- the concepts underlying the classification, 
- the capital market model underlying the simulation together with some product specific issues,

- some scientific challenges that are a consequence of experiences made in the first years of the classification.

\section{Typical private pension products offered in Germany}

The variety of offered private pension products greatly influences the classification effort, both from a conceptional point of view and from the purely technical issue of coding the algorithms. We therefore give a short and incomplete survey of the main types of private pension products offered at the German market that are eligible for state bonuses or tax advantages.

In former times, the market for private pension products in Germany was dominated by traditional life insurance contracts with surplus participation offering a high degree of safety and by unit-linked fund investments offering a high return potential, but leaving the investment risk with the policy holder. During recent years and particularly motivated by the current low interest rate phase, the diversity of state subsidized private pension products has enormously increased. Besides the above mentioned two standard types, there is nowadays a wide range of hybrid products that combine fund investment with guarantee components, new forms of the participating life insurance, savings plans, or index participations, just to name the main families. Most of these products also have variants containing a money-back guarantee, i.e. their contract value $V(T)$ at the end of the accumulation period exceeds the sum of contributions paid in by the customer and potential bonuses assigned by the state. This property is a strict requirement for the products to fall in the range of the so-called Riester-Rente, the main German bonus scheme for private pension products. All German employees and their spouse are eligible for subsidy under the Riester-Rente scheme.

To give the reader a survey on the main characteristics of all those product classes, we will roughly describe them below. While in the actual simulation we are working on the basis of monthly premium payments by the policy holder, it will here be beneficial for simplicity of the presentation that we assume annual contributions by the policy holder only in this work.

In the following, $B(t)$ denotes the annual premium that the policy holder contributes at the beginning of year $t$ until the end of the accumulation period which can - again for simplicity — be identified with the beginning of the retirement phase of the policy holder. We make the usual convention that the contract starts at $t=0$ with the first contribution. We also 
introduce $V(t)$ to be the policy value at time $t, N(t)$ the net savings premium after deduction of initial costs $C(t)$ from the contribution $B(t)$, i.e.

$$
N(t)=B(t)-C(t) .
$$

Further costs that are annually deducted from the contract value are denoted by $C_{a}(t)$. Note that the costs can also vary in their functional form over time. A typical feature causing this is the so-called Zillmerung which assigns the main parts of the compensation for the agent selling the product to the first five years of the contract.

Traditional life insurance with surplus participation. We start with the classical pension product where each initial contribution $B(t)$ is decomposed into costs $C(t)$ and the net savings $N(t)$. The complete contract value is then invested in the actuarial reserve fund (ARF) of the insurer which is a special fund made up of various asset classes. At the end of the year, the contract value is assigned a surplus participation $i(t)$ based on the performance of the ARF (and of saved costs, mortality gains and further reserves gained from earlier periods and/or the (assigned) differences between market and book value of the assets in the ARF). This surplus participation has to exceed the promised guarantee rate at the start of the contract $i_{G}(0)$ which currently is $0,9 \%$ in Germany, i.e. $i(t) \geq i_{G}(0)$. The contract value thus evolves according to

$$
V(t)=\left(V(t-1)-C_{a}(t)\right)(1+i(t))+N(t),
$$

where bonuses $R(t)$ from the state may also be granted. As they depend on both the actual contribution $B(t)$ and the personal situation of the policy holder (married, children, income, ...), we omit them here for simplicity (although this can be quite a rough simplification, it is in line with the requirements for the simulation given by the German Ministry of Finance).

There also exist variants where the guarantee rate $i_{G}(0)$ is reduced, sometimes even to zero. This variant is often called neo classic or capital efficient classic. Further, there are variants where a part of the surplus is invested into a fund.

However, the main challenge in the current interest rate environment independent of the product type - is the money-back guarantee

$$
V(T) \geq \sum_{t=0}^{T-1} B(t) .
$$


Index participations. Index participations allow the policy holder to benefit from a positive evolution of a stock index although all the (net savings) contributions of the policy holder are invested into the ARF. However, that part of the annual surplus that is not needed to ensure the moneyback guarantee can be used to buy an option on the annual performance of an index. To obtain a significant chance for additional return, typically an option with a high leverage is used. An example which is popular is a so-called locally capped and globally floored cliquet-option with a payoff at time $t+1$ of

$$
Z(t+1 ; C)=\tilde{V}(t)\left(\sum_{i=1}^{12} \min \left(C, \frac{S\left(t+\frac{i}{12}\right)-S\left(t+\frac{i-1}{12}\right)}{S\left(t+\frac{i-1}{12}\right)}\right)\right)^{+} .
$$

Here, $C$ is called the index cap and is determined by the money available for buying the option. $\tilde{V}(t)$ denotes the contract value at time $t$ after all costs are deduced, $S(t)$ the value of the index at time $t$. At time $t+1$ the payment $Z(t+1)$ is added to the contract value. Variants using other option types exist at the market.

The particular feature of option type payoffs as in Equation (4) is that on the one hand they provide the possibility of high returns on the contract value by leveraging the contract value (there have been annual returns realized in practice of about $20 \%$ ) while on the other hand the downside risk is limited by the option price paid. Compare this to either a full (!) investment of the contract value in the fund underlying the index which can lead to a higher return but does not provide any guarantee, or to the investment of the surplus in the index which can only generate a high return on the surplus and not (!) on the whole contract value. Of course, an option payment of zero does also appear quite often.

Unit-linked life insurance. Here, the net savings contribution of the policy holder is invested into a fund. The evolution of the contract value is given by

$$
V(t)=\left(V(t-1)-C_{a}(t)\right)\left(1+r_{F}(t)\right)+N(t)
$$

with

$$
1+r_{F}(t)=\frac{F(t)-F(t-1)}{F(t-1)}
$$

where $F(t)$ denotes the value of the fund at time $t$. Typically, the investment risk stays with the customer. To highlight this feature, we have used a 
notation of the fund return that differs from $i(t)$ which has been the return in the traditional life insurance product with surplus participation.

There are also special variants of unit-linked products including guarantees. They are then summarized under the name hybrid products.

Hybrid products. The term hybrid product is assigned to those products where money is allocated to both a guarantee component and to a pure investment component. Hybrid products mainly differ in the way how the given guarantee (such as the money-back-guarantee or the guarantee of a fixed percentage of all contributions) is generated. Typically, a life insurer uses the ARF as the guarantee component. A fund provider instead has to rely on the capital market and either uses a bond strategy, put options, or a sophisticated portfolio strategy such as a CPPI-strategy (see e.g. [2], [6]). Hybrid products are also distinguished according to the nature of their investment strategy. There are so-called static hybrids where the allocation of the net savings contribution to the guarantee and to the investment part of the product stays constant over time. In dynamic hybrids this strategy is state and time dependent. According to the funds used there exist twoand three-fund hybrids. In the two-fund setting there typically exist a strict guarantee part such as the ARF and a second fund that has a lower guarantee (a usual ingredient is a fund that guarantees $80 \%$ of its initial value and which is typically hold for one month or one year). The three-fund version in addition contains the possibility to invest in a fund that does not promise any kind of guarantee.

Savings plans. A savings plan is typically offered by banks. Here, the full contributions of the customer are added to the contract account. At the end of the year a fixed, a variable, or a combined interest rate $i(t)$ is assigned to the contract value. This is often a market rate such as the rate for 10 year bonds minus a predefined deduction. Thus, costs are introduced in an indirect way. The evolution of the contract is thus given by

$$
V(t)=V(t-1)(1+i(t))+N(t) .
$$

Due to a possible non-negativity condition or to a bonus scheme, the determination of the interest rate $i(t)$ can also contain non-linearities. 
More products. There are many more different products which we do not describe here as they do not constitute a significant part of the German pension market. Of course, they still have to be simulated and classified. As they often also have features such as e.g. a so-called intelligent volatility control, a complicated fund investment strategy, separate treatment of state bonuses and of contributions made by the policy holder, they often cause a large effort with respect to the actual implementation of the simulation procedure.

\section{Aspects of chance-risk classification concepts}

There are well-known relations between chance and risk as e.g. expressed in a mean-variance or a mean-standard-deviation diagram for the return of an asset. The use of those diagrams is a natural basis for setting up chance-risk classes (CRC). However, we first have to decide about the form of the measures that stand for chance/return and for risk.

As the prototypical customer pays the fixed contribution of 100 Euro every months, the use of the (continuous) yield $Y$ that is determined from the mean of the 10000 simulated contract values at the end of the accumulation period is the choice for the chance measure. We thus have to solve

$$
100(1+Y / 12) \frac{(1+Y / 12)^{12 T}-1}{Y / 12}=\frac{1}{10000} \sum_{i=1}^{10000} V^{(i)}(12 T)
$$

where $V^{(i)}(12 T)$ denotes the final contract value in the $i$ th simulation run. Note that we take the yield corresponding to the average of the simulated realizations of the accumulated final contract value and not (!) the average yield. The reason for this is that we are thus in line with the definition of the yield of a zero bond and with the expected value of a stock.

As a surprisingly high return constitutes no risk at all for a pensioner, we solely concentrate of the downside risk. A number which is based on the philosophy of the expected short fall measure is obtained using the 2000 worst simulated final contract wealth and then calculate the corresponding yield as in Equation (8) where of course, we have to divide by 2000 only. We will call this solution $X$.

Having decided on the chance and on the risk measures, our next task is to determine the five different chance-risk classes. For this we define reference portfolios which mirror the intended behavior of the class. For example, the reference portfolio attached to CRC 1 solely consists of AAAbonds which mature at the end of the accumulation period $T$. The portfolio 
attached to CRC 5 invests in our prototypical fund with a volatility of $20 \%$. The reference pairs of the chance measure and risk measure points are then put into a diagram that displays the chance measure on the y-axis and the (negative (!) of the) risk measure on the x-axis. This way of display ensures that moving to the top (in terms of a higher chance) and moving to the left (in terms of a lower risk) is desirable. Different product types and their chance-risk pairs are plotted in Figure 1.

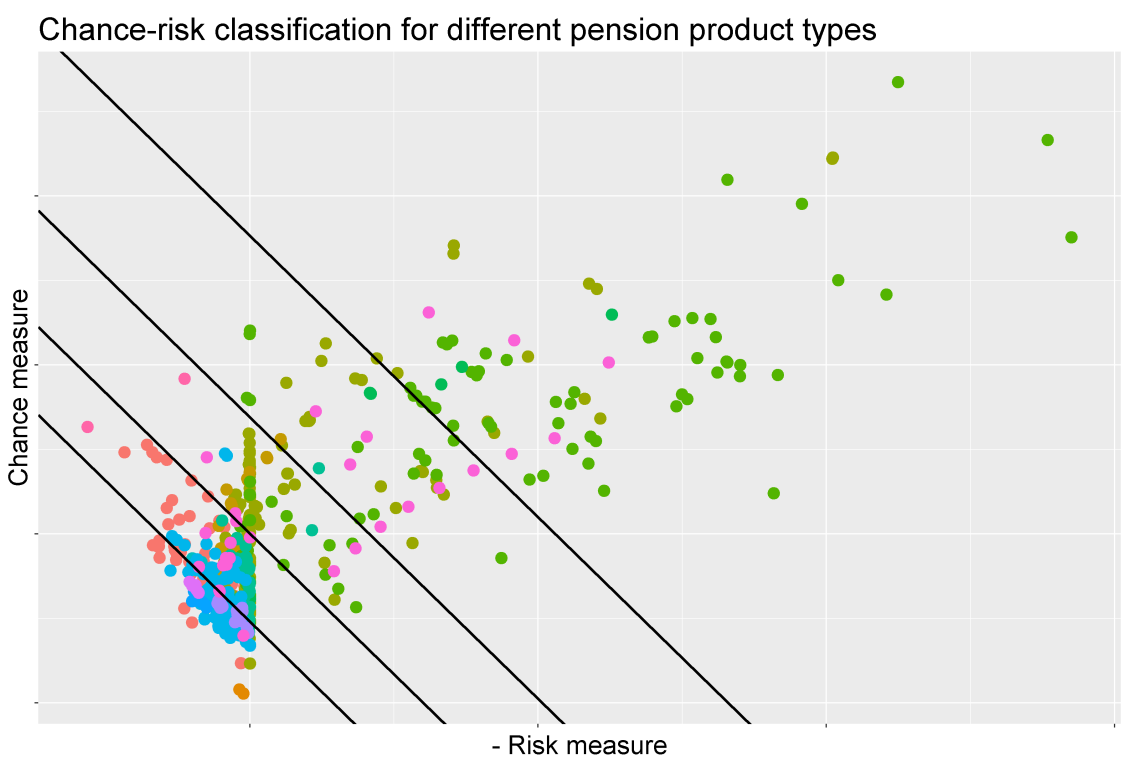

Fig. 1. Chance and risk pairs for classified products $(12 \mathrm{~T}=30)$.

To separate the different CRC we draw lines with a slope of -1 lying in the middle between the points that represent the reference portfolios. The justification for the slope of -1 of the separating lines lies in the relation between the reference portfolios and the actually offered products. For the reference portfolios, the chance and the risk measure are calculated based on the simulated wealth at the end of the accumulation period without considering any costs. Due to the similar, mainly linear form of both the chance and the risk measure, the actual costs reduce them both in a similar, typically parallel way for real-life pension products. Of course, this parallelism is slightly disturbed when the money-back guarantee gets binding. 
There is a clear criticism for this way of constructing the areas for the chance-risk classification. Leaving aside the influence of the money-back guarantee, it seems that a product can stay in the same chance-risk class when the costs are arbitrarily increased. However, increasing costs will lead to an increase in the reduction in yield, the second quantity besides the chance-risk class that is shown to the customer. And of course, a high reduction in yield will make a product unattractive to the customer. So, the reduction in yield helps to force the product provider to offer products with as few costs as possible.

\section{Remark 3.1. Why are the values of the chance and the risk mea- sure not shown to the customer?}

As already mentioned, only the CRC of a product is shown to the customer as the result of the simulation process. Thus, the customer does not get the full information that is generated. However, there are good reasons for that. The main reason is that the primary task of the whole simulation of the performance of the products in the accumulation phase is the classification in the different CRC. It is therefore the relative performance among the different products that is sufficient. Although the real-life performance should be mirrored as close as possible, an exact image cannot be created due to e.g.

- differences in the time discretization between real-life and model simulation (in the classification process, all calculations and simulations have to based on a monthly scale why in real-life there are many advanced products that use weekly or daily reallocation of the portfolios),

- only approximate knowledge of the reallocation algorithm (as the exact form of the allocation algorithm and in particular strategic decision plans of the product providers are often proprietary knowledge, we receive only simplified forms of both),

- the worst-case principle (i.e. using upper bounds) that has to be applied with regard to variable costs and that does not allow to consider the possibility of kick backs.

All these reasons might lead to an under performance of the products in the simulation in absolute terms. In relative terms between the different products, however, this is mainly not the case.

On the other hand, the way of calculation of the prices of options that are ingredients of various products (such as e.g. index participations) 
assumes a liquid market. In real-life, those prices can be significantly higher due to the fact that full liquidity and market efficiency for such option types is not given. This might lead to significantly higher market prices than model prices. Thus, those products might perform worse in the real markets than in the simulations.

It has therefore been decided that the CRC and the reduction in yield are the only informations passed on to the customer that are generated on the basis of the stochastic market model.

\section{Capital market model and simulation of important product ingredients}

The choice of the capital market model is crucial for both the simulation effort and also for the realism of the classification. As for the majority of existing pension contracts bonds are the most important investment asset and as the duration of the contract is long, we decided to choose a two-factor Hull-White model stated in the so-called $G 2-++$ formulation (see [3]). The model has the advantage of being a well-tested and applied model. Also, as a two factor model, it avoids the problem of perfect correlation between short- and long-term interest rates. Further, as it admits a normally distributed short-rate behavior, it has the potential to model negative interest rates. And finally, it allows for a perfect initial calibration to the bond market.

The model is given by the equations

$$
\begin{aligned}
& d x(t)=-a x(t) d t+\sigma d W_{1}(t), \\
& d y(t)=-b x(t) d t+\eta\left(\rho d W_{1}(t)+\sqrt{1-\rho^{2}} d W_{2}(t)\right), \\
& r^{*}(t)=x(t)+y(t)+\zeta(t)
\end{aligned}
$$

describing the dynamics of the short rate $r(t)^{*}$ under a risk-neutral measure $Q . W_{i}(t)$ are independent one-dimensional Brownian motions. The function

$$
\begin{aligned}
\zeta(t)=f^{M}(0, t)+\frac{\sigma^{2}}{2 a^{2}}\left(1-e^{-a t}\right)^{2}+ \\
\quad \frac{\eta^{2}}{2 b^{2}}\left(1-e^{-b t}\right)^{2}+\rho \frac{\sigma \eta}{a b}\left(1-e^{-a t}\right)\left(1-e^{-b t}\right)
\end{aligned}
$$

contains the model parameters that are calibrated to market prices of swaptions, while the initial market forward rate curve $f^{M}(0, t)$ is obtained from the Nelson-Siegel-Svensson curve of the Deutsche Bundesbank. 
As for the classification purposes we have to simulate all relevant processes under a real-world measure $P$, we have to add a risk premium to obtain the final short rate process

$$
r(t):=r^{*}(t)+\lambda_{r}(t) .
$$

To determine it, we use the annual predictions by the OECD on the expected future development of the interest rate market. We here do not go into further technical details.

On the stock side, we use a generalized Black-Scholes model given by

$$
S(t)=s_{0} \exp \left(\int_{0}^{t} r(s) d s+\left(\lambda_{S}-0.5{\sigma_{S}}^{2}\right) t+\sigma_{S} W(t)\right)
$$

for modeling the dynamics of a reference stock index. Here, the onedimensional Brownian motion $W(t)$ can also be correlated to $W_{i}(t)$.

Based on an analysis of the EuroStoxx 50, we have chosen the parameters $\lambda=0.04$ and $\sigma=0.2$ to give $S(t)$ comparable dynamics. If a product is based on another index or stock, then we assume that it has the dynamics as our basic index $S(t)$, but with individual $\sigma$ and a risk premium of $\lambda$ determined by

$$
\lambda=\lambda_{S} \frac{\sigma}{\sigma_{S}} .
$$

Remark 4.1. Why not using a more general stock price model?

Of course, one can think about introducing jumps or even Lévy-type models for the evolution of the stock index. However, the choice of the market model had to be a compromise between generality, stability of calibration and the market standards. Note that for some products, we need option prices, continuous-time approximations for discrete formulas and other features where more complicated models do not offer stable or unique solutions (think about e.g. option prices in incomplete markets!). Due to the long running contracts and the dominance of bond investments in life insurance, our preference was to consider (at least) a two-factor model for the short rate and not to introduce a more complicated stock price model.

Having specified the basic processes for our capital market, we now have to come up with the simulation of some important components that are crucial for many products. The most prominent of those components is the ARF of an insurance company and - as a consequence of its annual performance - the surplus declaration mechanism. 
How to simulate the surplus declaration in a participating life insurance contract? Our modeling approach is based on the following ideas: We assume an insurer to buy bonds of duration $d$ at par every year. Thus, the main risk is the re-investment risk when those bonds mature. Otherwise, we know their annual coupons precisely in advance. Let, therefore be $k_{d}(t-i)$ be the coupon rate of a $d$-year bond bought at time $t-i$. It will pay out those coupons at years $t-i+1, \ldots, t-i+d$. Assuming a stationary state of the insurance company in the sense that the number of bonds hold are constant over the years, we arrive at a mean annual bond return of

$$
R_{B, d}(t)=\frac{1}{d} \sum_{i=1}^{d} k_{d}(t-i)
$$

Further, we assume a constant proportion investment strategy over time, i.e. the insurer always rebalances the holdings at time $t$ such that the fraction invested in stocks (or a comparable fund) equals $\psi \in[0,1]$.

To model the smoothing algorithm for the surplus rates over time applied by a life insurance company, we assume that the geometric average over the returns of the last three years (including the current one) is the basis for the surplus declaration. It is given by

$$
R(t)=\sqrt[3]{\prod_{i=0}^{2}\left(\psi \frac{F(t-i)}{F(t-i-1)}+(1-\psi)\left(R_{B, d}(t-i)+1\right)\right)}-1 .
$$

Based on this, we assign the following surplus rate to the actuarial reserve fund

$$
g(t+1)=i_{G}(0)+\left(0.9 *(R(t)-K)-i_{G}(0)\right)^{+},
$$

where $i_{G}(0)$ is the guarantee rate which has been valid when entering the contract. Note that the second term on the right hand side contains the minimal part (by law $90 \%$ ) of the returns $R(t)$ after capital costs $K$ that the insurer has to assign to the surplus rate, in case that this part is positive.

Our algorithm is a suggestion to capture the freedom of the insurer to decide about direct assignments of gains to the individual contracts or to a collective bonus account without (!) directly introducing this feature. Introducing all kinds of accounts and legal aspects goes beyond the scope of this paper. However, for a detailed treatment of the use of bonus accounts, reserve accounts and liabilities, we refer to [5]. 
How to generate guarantee mechanisms? Given the above relation for the surplus rate, we can at least assume a return of $i_{G}(0)$ each year. It is thus the classical tool for a life insurer to generate guarantees, simply by contract agreement, not so much by the use of the capital market. Therefore, the actuarial reserve fund is also used by insurers in hybrid products. However, depending on the construction of the hybrid product, the hybrid product will attain the full surplus or only a part of the surplus that the holder of a classical product with participation receives.

If however the actuarial reserve fund can not be used (for e.g. contractual reasons) or if the provider of a pension product simply does not have an actuarial reserve fund (think of a bank or an investment company) then CPPI-type strategies using bonds or option based strategies are popular alternatives.

How are de-risking strategies implemented? Reducing the investment risk with approaching end of the accumulation phase is a typical strategy to avoid big losses when there is not a long time remaining to correct them. While there are small differences in the ways this is implemented in various products offered at the market, the main form consists of a linear reduction of stock-type parts down to zero close to the end of the accumulation phase.

\section{Scientific challenges and outlook}

Of course, the whole project of chance-risk classification of publicly available pension products is not a playground for scientific experiments. On one hand, well established models and methods should be used while on the other hand, it needs a very efficient implementation of the simulation process of a wide range of diverse products. Thus, there is always room for improvement, in particular as also new products will enter the scene.

The simulation of some products needs a particularly high computational effort. Given that we have to simulate 10000 paths of the evolution of the contract value over 40 years, efficiency can be greatly improved if regularly appearing tasks can be accelerated.

Improved Monte Carlo algorithms for pricing options in an index participation. A prototypical example of both high computational effort per simulation path and of accelerating regularly appearing tasks is the calculation of the implied monthly cap on the index return in index participation products. Here, we refer the reader to Korn, Temocin and Wenzel 
[8] where a completely new control variate approach for pricing cliquet-style options is developed. It rests on the use of a control variate that has been derived using the central limit theorem. More precisely, by identifying the twelve capped monthly returns

$$
R^{c}(i)=\min \left\{\frac{S(t+i / 12)-S(t+(i-1) / 12)}{S(t+(i-1) / 12)}, c\right\}, i=1,2, \ldots, 12,
$$

in the cliquet option payment as independent and identically distributed random variables in the Black-Scholes setting, we use the approximation

$$
\left(\sum_{i=1}^{12} R_{i}\right)^{+} \approx X^{+}
$$

with $X \sim N\left(12 E\left(R_{i}\right), 12 \operatorname{Var}\left(R_{i}\right)\right)$. This is motivated by the central limit theorem (although 12 might appear to be quite small for the use of an asymptotic result). While the approximate option price $E\left(\exp (-r) X^{+}\right)$ (under the risk-neutral measure) can be obtained as an explicit analytical formula and is surprisingly close to the exact option price, it is not close enough to replace the option price fully as an approximation. However, using this approximate price as a control variate delivers a great variance reduction in the Monte Carlo simulation.

The resulting control variate method can numerically outperform the standard semi-closed formula solution as given by Bernard and Li [1]. Even more, it can be extended to more general model frameworks such as the Heston model and also to other types of options used in index participations such as the asymmetric participation option (see again [8]). The latter is not dealt with by Bernard and $\mathrm{Li}[1]$.

\section{The projection of general assets to the two basic asset classes.}

Many investment positions hold by life insurance companies are no pure bond or stock products. Examples are defaultable bonds, investment in buildings, wind parks, just to name a few. They typically contain aspects of a bond-type and also of a stock-type investment. Also, derivatives such as stock options have a mixed character. The projection of such assets in a suitable way to the two basic asset classes is an important issue for the realistic simulation (and performance) of the actuarial reserve fund. A corresponding decomposition algorithm that assigns an investment product must be simple and also convincing from a conceptual point of view. Therefore, the simulation model for official classifications has recently been extended to allow for a more complex structure of the ARF. 
Effects in the evolution of the yield curve. The so-called yield curve for zero bonds with maturity $x$ is defined as the equivalent constant interest rate $y(x)$ that one receives when buying a zero bond now for the price of $P(0, x)$ and holding it until maturity $x$. The most popular forms of the function $y(x)$ are a normal yield-curve if it is increasing and concave, an inverse yield curve if it is decreasing and convex, and a humped yield curve if it contains exactly one interior maximum. Of course, further forms of yield curves are possible, and some also have names.

Simple short rate models such as the one-factor Vasicek model have a tendency to produce a higher fraction of inverse term structures with ongoing time. The reason for this is based on the following two facts (see e.g. [4]):

- For large values of $x$ in the Vasicek model we have that $y(x)$ converges to a fixed value $\bar{y}$.

- The stationary distribution of the short rate $r(t)$ has a median that is bigger than $\bar{y}$.

Thus, due to the fact that the current value of the short rate determines the form of the yield curve, we will asymptotically observe more than $50 \%$ of inverse or humped yield curves in the Vasicek model. This follows from the fact that for initial short rate values above $y$ the yield curve in the Vasicek model can only be inverse or humped (see again Chapter 8 in [4]).

The two-factor Hull-White variant that we are using has a similar behavior, but the relations are more subtle and in particular also depend very much on the initial yield curve that we obtain from calibrating the model to the yield curve of the Deutsche Bundesbank. Obtaining a simple relation between the starting yield curve and the distribution of future forms of the yield curve at some fixed time point is therefore an obvious research challenge.

The form of chance-risk curves. An effect that is at first sight surprising is the dependence of the form of the chance-risk curve on its ingredients. Knowing the typical form of a classical mean-standard deviation diagram, we would also expect a typical form for the chance-risk curve as in Figure 2. We have plotted the chance measure on the y-axis and have plotted the risk measure on the $\mathrm{x}$-axis, but in inverted order. This is done to have a high risk on the right hand side of the figure (as in the usual risk-return plots). One can however show that in a simple Black-Scholes setting for a high risk premium of stock investment, for long time to maturity $T$ or for 


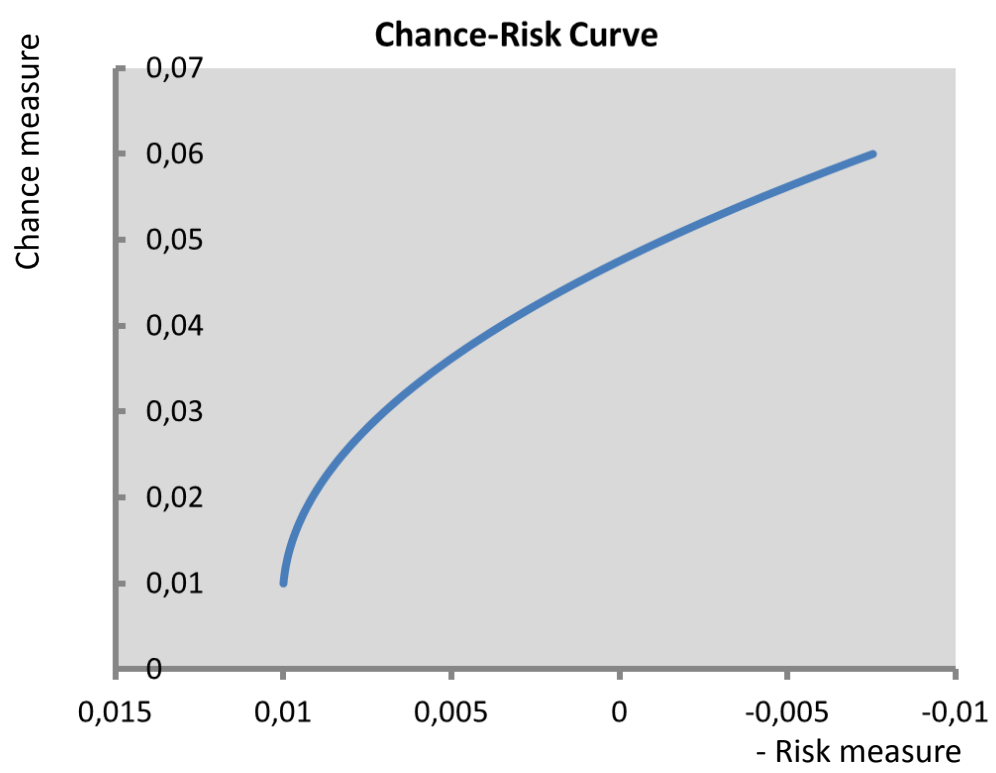

Fig. 2. A form of a chance-risk-curve similar to a mean-standard-deviation curve.

a low volatility $\sigma$ there can occur different forms. Thus, to obtain explicit descriptions of the form of the chance-risk curve in dependence of the input parameters and ingredients (such as the initial yield curve) is another desirable goal.

More challenges. Due to the low interest rate environment, we expect pension products to use even more complicated investment and guarantee strategies in the future. Products that use a daily asset reallocation strategy already exist. Other products contain a volatility control that uses the volatility estimation on the basis of daily asset prices. Of course, for every such product, it is a challenge to find approximation algorithms that are based on our monthly discretization. Further challenges will appear with every new generation of products.

\section{References}

1. Bernard, C., Li, W.V.: Pricing and hedging of cliquet options and locally-capped contracts. SIAM J. Fin. Math. 4, 353-371 (2013). 
2. Black, F., Jones, R.: Simplifying portfolio insurance, Journal of Portfolio Management 14, 48-51 (1987).

3. Brigo, D., Mercurio, F.: Interest Rate Models: Theory and Practice. Springer Finance, Berlin (2001).

4. Desmettre, S., Korn, R.: Moderne Finanzmathematik - Theorie und praktische Anwendung, Band 2. To appear: Springer Spektrum, Heidelberg (2018).

5. Kling, A., Richter, A., Russ, J.: The Interaction of Guarantees, Surplus Distribution, and Asset Allocation in With Profit Life Insurance Policies, Insurance: Mathematics and Economics 40, 164-178 (2007).

6. Korn, R.: Moderne Finanzmathematik - Theorie und praktische Anwendung, Band 1. Springer Spektrum, Heidelberg (2014).

7. Korn, R., Korn, E., Kroisandt, G.: Monte Carlo Methods and Models in Finance and Insurance. Chapman \& Hall/CRC Financial Mathematics Series, Boca Raton, USA (2010).

8. Korn, R., Temocin, B., Wenzel, J.: Applications of the Central Limit Theorem for Pricing Cliquet-Style Options. European Actuarial Journal, doi=10.1007/s13385-017-0158-y (2017). 